\title{
INFLUENCE OF INTERMOLECULAR DIPOLAR INTERACTION ON SPIN-LATTICE RELAXATION OF THE THREE-PROTON GROUP
}

\author{
G. ŚLÓSAREK \\ Institute of Bioorganic Chemistry, Polish Academy of Sciences \\ Noskowskiego 12/14, 61-704 Poznań, Poland
}

(Received October 15, 1990)

\begin{abstract}
Spin-lattice relaxation of a system of three-proton group and a single, motionless spin is analysed. Only the dipole-dipole interaction is taken into account. The mathematical description is compared with the previous results known from literature.
\end{abstract}

PACS numbers: 76.60.Es

Spin-lattice relaxation process of the three-proton group in solids has been studied for many years, while NMR relaxation experiments have turned out to be a powerful tool in the analysis of the molecular dynamics of such a system. In most cases quantum aspects of this kind of molecular reorientations have been considered. Tunnelling, which is influenced both by intra- and intermolecular interactions, reflects the molecular environment of a given group. However, the classical description of the reorientation in the high temperature range is also of some importance, particularly in the case, when the three-spin group plays an important role in the crystal lattice phase transition (see e.g. [1]).

In the system of three-proton groups in a solid the most effective mechanism of proton spin-lattice relaxation is the dipole-dipole interaction between the nuclei within the group and between these protons and any other nucleus. In the first case one considers a system of isolated three-proton groups, where only a homonuclear interaction is taken into account [2-5]. In the other case the homonuclear as well as the heteronuclear interaction is analysed. The system of pairs of the three-proton groups has been already described [7-10]. This short communication presents the analysis of the change of the relaxation rate caused by the dipole-dipole interaction between the protons within the group and a single, motionless proton from the same or another molecule. A similar system has been analysed before by Michel and Rössiger [10]. Below the results of both analyses are compared. 


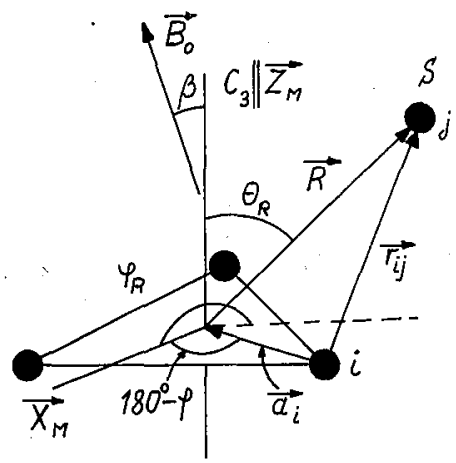

Fig. 1. Geometry of the spin system. $\left(X_{M}, Y_{M}, Z_{M}\right)-$ molecular frame, $B_{0}$ is parallel to the $Z$ axis of the laboratory frame.

The geometry of the spin system under consideration is presented in Fig. 1. In general, it corresponds to the spin arrangement analysed in [10]. It is assumed that three protons form a triangle with the $C_{3}$ symmetry axis. Distances between the nuclei in the system are described by vectors $r_{i, j}, \boldsymbol{R}$ and $\boldsymbol{a}_{i}$. The dipole-dipole interaction under consideration depends on $r_{i, j}$, which changes its length and direction in time because of the rotation of the group. However, the description of the time dependence of $r_{i, j}$ is not very complicated, while $r_{i, j}$ is a sum of the constant vector $\boldsymbol{R}$ (it is assumed that spin $S$ is motionless) and vector $\boldsymbol{a}_{i}$, whose time dependence in the molecular frame is described by a function $\varphi(t)$. The coordinates of the spin $S$ in the molecular frame are $R, v_{R}, \varphi_{R}$. The angle between the symmetry axis $C_{3}$ and the $Z \| B_{0}$ direction in the laboratory frame equals $\beta$.

According to Abragam [11] the spin-lattice relaxation rate equals:

$$
T_{1}^{-1}=\frac{3}{2} \gamma^{4} h^{2} I(I+1)\left[J^{(1)}(\omega)+J^{(2)}(2 \omega)\right]
$$

where

$$
\begin{gathered}
J^{(n)}(\omega)=\int_{-\infty}^{\infty} G^{(n)}(\tau) \exp (-\mathrm{i} \omega \tau) \mathrm{d} \tau \\
G^{(1)}(\tau)=\sum_{i, j}\left\langle b_{i j}(\tau) l_{i j}^{*}(0)\right\rangle \\
G^{(2)}(\tau)=\sum_{i, j}\left(c_{i j}(\tau) c_{i j}^{*}(0)\right\rangle, \\
b_{i j}=\sqrt{2 \pi / 15} \frac{2}{r_{i j}^{3}} Y_{2-1}\left(\vartheta_{i j}, \varphi_{i j}\right), \\
c_{i j}=\sqrt{2 \pi / 15} \frac{4}{r_{i j}^{3}} Y_{2-2}\left(\vartheta_{i j}, \varphi_{i j}\right)
\end{gathered}
$$


$\left(r_{i j}, \vartheta_{i j}, \varphi_{i j}\right.$ - coordinates of the vector $r_{i j}$ in the laboratory frame). While $r_{i j}=\boldsymbol{R}+\boldsymbol{a}_{i}$, functions $b_{i j}$ and $c_{i j}$ can be transformed using the following formula [12]:

$$
\frac{1}{r^{L+1}} Y_{L M}(\Omega)=\sqrt{4 \pi /(2 L) !} \sum_{l_{1}, l_{2}, l_{2}-l_{1}=L} \frac{\left(2 l_{2}\right) !}{\left(2 l_{1}+1\right) !} \frac{r_{1}^{l_{1}}}{r_{2}^{l_{2}+1}}\left\{Y_{l_{1}}\left(\Omega_{1}\right) \otimes Y_{l_{2}}\left(\Omega_{2}\right)\right\}
$$

where $r_{1}=a$ and $r_{2}=R, \Omega_{1}$ and $\Omega_{2}$ are the angular coordinates of $a$ and $\boldsymbol{R}$ in the laboratory frame. While only the time dependent part of the functions $b_{i j}$ and $c_{i j}$ are taken into account the first term of the series (7), which is time dependent, is that with $l_{1}=1$ and $l_{2}=3$. The functions $b_{i j}$ and $c_{i j}$ are transformed to molecular frame according to the known procedure [12]:

$$
\left\{Y_{l_{1}}\left(\Omega_{1}\right) \otimes Y_{l_{2}}\left(\Omega_{2}\right)\right\}_{L M}=\sum_{M} D_{M M}^{L}(\alpha, \beta, \gamma)\left\{Y_{l_{1}}\left(\Omega_{1}\right) \otimes Y_{l_{2}}\left(\Omega_{2}\right)\right\}_{L M}
$$

where $\alpha=\gamma=0$.

After calculations of correlation functions $G^{(n)}(\tau)$ and the spin density functions $J^{(n)}(\omega)$, one obtains the relaxation rate, which equals:

$$
T_{1}^{-1}=\frac{9}{256} \gamma_{\mathrm{H}}^{4} h^{2} \frac{r^{2}}{R^{8}}\left(\frac{B \tau_{\mathrm{c}}}{1+\omega_{\mathrm{H}}^{2} \tau_{\mathrm{c}}^{2}}+\frac{C \tau_{\mathrm{c}}}{1+4 \omega_{\mathrm{H}}^{2} \tau_{\mathrm{c}}^{2}}\right)
$$

where

$$
\begin{array}{r}
B=\left(1-\cos ^{4} \beta\right)\left(a^{2}+b^{2}\right)-2\left(1-\cos ^{2} \beta\right)^{2} a b \cos 4 \varphi_{R} \\
+4(a c+b d) \sin \beta \cos ^{3} \beta \cos \varphi_{R} \\
+4(a d+b c) \sin \beta \cos \beta\left(\cos ^{2} \beta-1\right) \cos 3 \varphi_{R} \\
+6(a e+b e) \sin ^{2} \beta \cos ^{2} \beta \cos 2 \varphi_{R}+\left(4 \cos ^{4} \beta-3 \cos ^{2} \beta+1\right)\left(c^{2}+d^{2}\right) \\
+2 c d\left(4 \cos ^{4} \beta-5 \cos ^{2} \beta+1\right) \cos 2 \varphi_{R} \\
+6(c e+d e) \sin \beta \cos \beta\left[2\left(\cos ^{2} \beta\right)-1\right] \cos \varphi_{R}+9 e^{2} \sin ^{2} \beta \cos ^{2} \beta \\
C=\left(1+6 \cos ^{2} \beta+\cos ^{4} \beta\right)\left(a^{2}+b^{2}\right)-2 a b \sin ^{4} \beta \cos 4 \varphi_{R} \\
-4 \sin \beta \cos \beta\left(3+\cos ^{2} \beta\right)(b d-a c) \cos \varphi_{R} \\
+4(b c-a d) \sin ^{3} \beta \cos \beta \cos \varphi_{R}-6(a e+b e)\left(1-\cos ^{4} \beta\right) \cos 2 \varphi_{R} \\
+\left(1-\cos { }^{4} \beta\right)\left(c^{2}+\dot{d}^{2}\right)-2 c d\left(1-\cos ^{4} \beta\right) \cos 2 \varphi_{R} \\
+12(c e+d e) \sin 3 \cos \beta \cos \varphi_{R}+9 e^{2} \sin { }^{4} \beta \\
a=(5 / 2)\left(3 \sin \vartheta_{R}-\sin 3 \vartheta_{R}\right), \\
b=(1 / 2)\left(\sin \vartheta_{R}+5 \sin 3 \vartheta_{R}\right) \\
c=-5\left(\cos \vartheta_{R}-\cos 3 \vartheta_{R}\right), \\
d=3 \cos \vartheta_{R}+5 \cos 3 \vartheta_{R},
\end{array}
$$




$$
e=\sin \vartheta_{R}+5 \sin 3 \vartheta_{R} .
$$

In the case of powder samples the averaged value of $T_{1}^{-1}$ equals:

$$
\begin{gathered}
T_{1}^{-1}=\frac{1}{4 \pi} \int_{0}^{2 \pi} \int_{0}^{\pi} T_{1}^{-1}(\beta) \sin \beta \mathrm{d} \beta \mathrm{d} \alpha \\
T_{1}^{-1}=\frac{9}{640} \gamma_{\mathrm{H}}^{4} \hbar^{2} \frac{r^{2}}{R^{8}}\left[\frac{B \tau_{\mathrm{c}}}{3\left(1+\omega_{\mathrm{H}}^{2} \tau_{\mathrm{c}}^{2}\right)}+\frac{2 C \tau_{\mathrm{c}}}{1+4 \omega_{\mathrm{H}}^{2} \tau_{\mathrm{c}}^{2}}\right]
\end{gathered}
$$

where

$$
\begin{array}{r}
B=(2 / 15)\left[6\left(a^{2}+b^{2}\right)-8 a b \cos 4 \varphi_{R}+6(a e+b e) \cos 2 \varphi_{R}\right. \\
\left.+6\left(c^{2}+d^{2}\right)+4 c d \cos 2 \varphi_{R}+9 e^{2}\right] \\
C=(4 / 5)\left[4\left(a^{2}+b^{2}\right)+6(a e-b e) \cos 2 \varphi_{R}+4\left(c^{2}+d^{2}\right)+\right. \\
\left.-8 c d \cos 2 \varphi_{R}\right]
\end{array}
$$

As it has been mentioned above the spin system under consideration is similar to that analysed by Michel and Rössiger [10]. These authors also assume that a single spin is motionless. The distance between the spins are described by a similar sum of vectors, but in [10] the origin of the molecular frame is different, which makes the calculations of the time dependence of the vectors length and direction very complicated. Because of that the authors present only the results of numerical calculations. There is no formula describing the angular dependence of $T_{1}$.

Michel and Rössiger discuss two models of the motion of the group-jumps between " $n$ " equivalent positions and pure rotational diffúsion. In the present communication only the first model is taken into accout. The correlation time of that motion equals $\tau_{\mathrm{c}}$.

According to the results of Michel and Rössiger (see e.g. Fig. 2 in [10]) the minimum of the temperature dependence of $T_{1}$ changes with the ratio of the length of vectors $a$ and $\boldsymbol{R}$, which is in contradiction to the results presented in this paper. According to equations (9) and (13) relaxation rate is only proportional to that ratio. However, the shape of the minimum of $T_{1}$ dependes on angles $\vartheta_{R}$ and $\varphi_{R}$, i.e. on the arrangement of the whole spin system under consideration.

\section{References}

[1] G. Ślósarek, A. Heuer, H. Zimmermann, U. Haeberlen, J. Phys. Condens. Maller. 1, 5931 (1989).

[2] E.O. Stejskal, H.S. Gutowsky, J. Chem. Phys. 28, 388 (1958).

[3] R.L. Hilt, P.S. Hubbard, Phys. Rev. 134, A392 (1964).

[4] J. Haupt, Z. Naturforsch. A 26, 1578 (1971).

[5] A.E. Zweers, H.B. Brom, Physica B 85, 223 (1977).

[6] D.E. Woessner, J. Chem. Phys. 42, 1855 (1965). 
Influence of Inlermolecular Dipolar Interaction on Spin-Latlice ... 743

[7] W.M. Zatsepin, T.N. Khazanovich, Zh. Eksp. Teor. Fiz. 74, 1800 (1978).

[8] G. Ślósarek, N. Piślewski, Acta Phys. Pol. A65, 315 (1984).

[9] G. Ślósarek, N. Piślewski, Acta Phys. Pol. A68, 717 (1985).

[10] D. Michel, V. Rössiger, J. Magn. Reson. 28, 235 (1977).

[11] A. Abragam, Principles of Nuclear Magnetism, Clarendon Press, Oxford 1961.

[12] D.A. Varshalovich, A.N. Moskalev, V.K. Hersonskij, K'vantovaya teoriya uglovovo momenta, Nauka, Leningrad 1975. 